Foro, Nueva época, vol. 19, núm. 2 (2016): 413-431

ISSN:1698-5583

http://dx.doi.org/10.5209/FORO.55382

\title{
EL TRABAJO TEMPORAL EN LA JURISPRUDENCIA COMUNITARIA ${ }^{1}$
}

\author{
Ignacio GonZÁLEZ DEL Rey RodRíGUEZ \\ Catedrático de Derecho del Trabajo y de la Seguridad Social \\ Facultad de Derecho de la Universidad de Oviedo \\ ignatium@uniovi.es
}

\section{UNIÓN EUROPEA, TRABAJO ATÍPICO Y CONTRATACIÓN TEMPORAL: LA DIRECTIVA 1999/70/CE RELATIVA AL ACUERDO MARCO SOBRE EL TRABAJO DE DURACIÓN DETERMINADA}

En las dos últimas décadas del pasado siglo xx la Comunidad o Unión Europea prestó una atención especial a la contratación laboral atípica, fundamentalmente con la doble finalidad de promover un tratamiento igualitario y no discriminatorio de los trabajadores en situación de mayor precariedad o vulnerabilidad, y de facilitar la libre competencia y circulación, evitando el denominado dumping social o competencia desleal en el establecimiento de condiciones de trabajo «a la baja», mediante una regulación más homogénea y común para todos los Estados miembros, aun de principios o de mínimos, en el marco del complejo equilibrio entre la seguridad y la flexibilidad, entre los intereses de los trabajadores y las empresas.

Así, en los años ochenta se intentaron adoptar instrumentos normativos en relación con el trabajo temporal y el trabajo a tiempo parcial. Primero de manera separada, atendiendo a las particularidades de uno y otro tipo de relación laboral. Después, ya en los años noventa, de manera conjunta para las distintas fórmulas de trabajo atípico, pero con atención diferenciada de los distintos problemas que en el mercado de empleo planteaban con carácter común dichas formas de trabajo, a saber: condiciones de

${ }^{1}$ El presente trabajo se enmarca en el Proyecto de Investigación «La jurisprudencia social del TJUE: de las libertades económicas y profesionales a la tutela del trabajo por cuenta ajena», dirigido por Joaquín García Murcia y financiado por el Ministerio de Economía y Competitividad (DER 2013-45781-P). 
trabajo, distorsiones de la competencia y protección de la salud y la seguridad de los trabajadores ${ }^{2}$.

En los primeros años noventa no llegó a culminar ninguna de estas iniciativas, adoptándose tan sólo dos Directivas relativas a la contratación laboral mucho menos ambiciosas. La primera, la Directiva 91/383/CEE del Consejo, de 25 de junio, por la que se completan las medidas tendentes a promover la mejora de la seguridad y de la salud en el trabajo de los trabajadores con una relación laboral de duración determinada o de empresas de trabajo temporal, atinente al núcleo más esencial y mínimo del Derecho de trabajo: la seguridad y la salud de las personas en el trabajo, y por ello, seguramente, de más fácil consenso. La segunda, la Directiva 91/533/CEE del Consejo, de 14 de octubre de 1991, relativa a la obligación del empresario de informar al trabajador acerca de las condiciones aplicables al contrato de trabajo o a la relación laboral, reguladora de derechos de información y no de condiciones de trabajo y empleo.

Hubo que esperar, no obstante, hasta bien avanzada esa década, y gracias al decisivo impulso del diálogo social y la negociación colectiva en el ámbito comunitario, reconocidos o reforzados en el Protocolo Social del Tratado de la Unión Europea de Maastricht, de 7 de febrero de 1992, para que vieran la luz las primeras Directivas reguladoras y protectoras de los derechos y las condiciones de trabajo y empleo de los trabajadores atípicos y precarios: la Directiva 97/81/CE, del Consejo, de 15 de diciembre de 1997, relativa al Acuerdo marco sobre el trabajo a tiempo parcial concluido por la Unión de Confederaciones de la Industria y Organizaciones Empresariales de Europa (UNICE), el Centro Europeo de la Empresa Pública (CEEP) y la Confederación Europea de Sindicatos (CES), y la Directiva 1999/70/CE, del Consejo, de 28 de junio de 1999, relativa al Acuerdo marco de la CES, la UNICE y el CEEP sobre el trabajo de duración determinada ${ }^{3}$.

A diferencia de otras Directivas comunitarias de contenido laboral, la Directiva 1999/70/CE, del Consejo, de 28 de junio de 1999, relativa al

\footnotetext{
2 Propuestas de Directivas del Consejo de 29 de junio de 1990, relativas a determinadas relaciones laborales, una en lo que respecta a las condiciones de trabajo y otra referida a las distorsiones de la competencia (DOCE, núm. C 224, de 8 de septiembre de 1990).

3 En estos años se adoptan también como Directivas relativas a Acuerdos marco europeos, la 1996/34/CE, del Consejo, de 3 de junio de 1996, relativa al Acuerdo marco sobre permiso parental; la 1999/63, del Consejo, de 21 de junio de 1999, relativa al Acuerdo sobre la ordenación del tiempo de trabajo de la gente del mar, y la 2000/79, del Consejo, de 27 de noviembre de 2000, relativa a la aplicación del Acuerdo europeo sobre la ordenación del tiempo de trabajo del personal de vuelo en la aviación civil.
} 
Acuerdo marco sobre el trabajo de duración determinada ${ }^{4}$, no tiene como presupuesto tanto una divergencia normativa entre Estados en materia de contratación temporal y una consecuente necesidad de armonización destinada a favorecer la libre circulación de trabajadores y una mayor transparencia y mejor competencia de las empresas en el mercado, como la necesidad de una mayor protección para los trabajadores temporales.

En este sentido, en su preámbulo y consideraciones generales, el Acuerdo marco parte de la caracterización del contrato de trabajo temporal como modalidad atípica frente al contrato ordinario de duración indefinida, aunque también útil como fórmula de trabajo más flexible para las empresas y necesaria en determinados sectores de actividad y ocupaciones, incluidas las de carácter estacional, y tiene por objeto la garantía del principio de igualdad y no discriminación de los trabajadores temporales, la mejora de la calidad del trabajo temporal, con exclusión expresa de la previsión social, y la prevención y proscripción del abuso en su utilización sucesiva o encadenada (cláusula 1$)^{5}$.

Atendiendo a su finalidad y objeto, la Directiva 1999/70/CE contempla fundamentalmente cuatro grupos de reglas sustantivas, relativas al principio de igualdad y no discriminación de los trabajadores temporales (cláusula 4), a la prevención del abuso y el fraude en la contratación de duración determinada (cláusula 5), a la promoción y estabilización de los trabajadores (cláusula 6), y a la relación con la representación colectiva en la empresa (cláusula 7). Los dos primeros grupos de normas constituyen el núcleo duro y más efectivo de la Directiva, y han sido claves en el fortalecimiento y mejora de las condiciones de trabajo y empleo de los trabajadores temporales en España, no sólo a partir de su transposición, sino también, y especialmente, a través de la jurisprudencia.

La aplicación jurisdiccional de esta Directiva, primero por parte del Tribunal de Justicia de la Comunidad Europea (TJCE) y después por su sucesor, el Tribunal de Justicia (TJUE), es destacable tanto en términos cualitativos como cuantitativos, y, como se dijo, ha tenido una especial incidencia en el ordenamiento interno español. Además, desde el punto de vista cualitativo, seguramente ha compensado la debilitación del interés o sensibilidad institucional de la Unión Europea por el trabajo y por los tra-

\footnotetext{
${ }^{4}$ Pese a que no resulte del todo ortodoxo, en el presente trabajo se aludirá indistintamente a la Directiva y al Acuerdo marco anexo a ella.

5 «La estabilidad en el empleo se concibe como un componente primordial de la protección de los trabajadores» (STJUE de 26 de noviembre de 2014, Mascolo, C-22, 61 a 63 y 418/13).
} 
bajadores precarios, a través de pronunciamientos que continúan la senda de la mejora de su protección ${ }^{6}$.

Desde el punto de vista cuantitativo, el Tribunal de Justicia ha adoptado alrededor de cuarenta resoluciones sustantivas en relación con la aplicación de la Directiva 1999/70/CE entre 2005 y 2016. Todas ellas a partir de cuestiones prejudiciales presentadas por los órganos de la jurisdicción de Estados miembros, salvo una dictada en recurso de incumplimiento (STJUE de 26 de febrero de 2015, Comisión Europea contra Gran Ducado de Luxemburgo, C-238/14), predominando las de origen griego, español e italiano, y resultando especialmente significativo que prácticamente todas ellas, salvo cuatro, se refieran a empleados de la Administración o del sector público.

Las cuestiones tratadas se refieren fundamentalmente a la conformidad de determinadas reglas nacionales con las cláusulas de la Directiva sobre igualdad y no discriminación de los trabajadores temporales (cláusula 4), y sobre prevención y evitación del abuso en el encadenamiento o sucesión de los contratos de duración determinada (cláusula 5). Sin perjuicio de algunos pronunciamientos relevantes, pero menos frecuentes, en relación con la delimitación del trabajo temporal o del contrato de duración determinada y el ámbito de aplicación de la Directiva (cláusulas 2 y 3), así como, más coyunturales, respecto de su transposición, puesta en práctica y efectividad en los Estados miembros (cláusula 8) ${ }^{7}$.

${ }^{6}$ Con el cambio de siglo, y seguramente como consecuencia de la significativa ampliación del número de Estados miembros de la Unión Europea y su diversidad y disparidad de políticas sociales, mercados de empleo, ordenamientos jurídicos y situaciones económicas, la política social comunitaria se ha visto ciertamente debilitada, y el diálogo social y la negociación colectiva europea dejaron de encontrar la favorable acogida que hasta entonces habían tenido en las instituciones y en los instrumentos normativos de la Unión, debilitándose también la propia predisposición negocial de los interlocutores sociales, con el agravante final de la crisis económica del cambio de década y los desfavorables tiempos para la «equiparación por la vía del progreso» en la protección de los trabajadores enunciado en la Carta Comunitaria de los Derechos Sociales Fundamentales de los Trabajadores, de 9 de diciembre de 1989. Así ha ocurrido, entre otros, con los Acuerdos marcos europeos sobre el teletrabajo, de 16 de julio de 2002, sobre el estrés ligado al trabajo, de 8 de octubre de 2004, y sobre el acoso y la violencia en el trabajo, de 26 de abril de 2007, no incorporados posteriormente a Directivas comunitarias. Y en otro sentido, con la Directiva 2008/104/CE, del Parlamento y del Consejo, de 19 de noviembre de 2008, relativa al trabajo a través de empresas de trabajo temporal, que no logró el previo acuerdo de los interlocutores sociales europeos y en la que prevalece la flexibilidad favorable a las empresas sobre la seguridad de los trabajadores, alejándose de sus precedentes en materia de trabajo temporal y a tiempo parcial.

7 Fundamentalmente, las SSTJCE de 7 de septiembre de 2006, Vasallo, C-180/04, y Marrosu, C-53/04; 15 de abril de 2008, Impact, C-268/06; 24 de junio de 2010, Sorge, C-98/09, y 22 de diciembre de 2010, Gavieiro, C-444/09 y C-456/09. 
Por lo demás, en la gran mayoría de los casos se estima la cuestión prejudicial, en el sentido de considerarse que la regla interna debatida se opone o es contraria a la Directiva europea o al Acuerdo marco anexo. Aunque en algunos se inste a los órganos judiciales remitentes a que verifiquen la posible justificación objetiva de las reglas cuestionadas, en su caso mediante la comprobación de la concurrencia de situaciones comparables, en relación con el principio de igualdad, o la existencia de medidas equivalentes alternativas en los ordenamientos nacionales en relación con la prevención y evitación de la utilización abusiva y sucesiva de la contratación temporal, y con los principios base de la Directiva, en particular, con el de su efectividad o efecto útil.

\section{II. ÁMBITO DE APLICACIÓN DE LA DIRECTIVA 1999/70/CE Y DELIMITACIÓN DEL TRABAJO TEMPORAL}

Conforme a la cláusula 2 de la Directiva 1999/70/CE, su ámbito de aplicación se refiere a los trabajadores sujetos a contratos temporales o de duración determinada, de acuerdo con el concepto adoptado en cada país, entendiéndose, no obstante, por tales, los contratos de trabajo «concertados directamente entre un empresario y un trabajador» cuyo fin esté «determinado por condiciones objetivas, tales como una fecha concreta, la realización de una obra o servicio determinado, o la producción de un hecho o acontecimiento determinado» (cláusula 3.1).

Se trata de contratos en los que desde el inicio se acuerda un término o momento extintivo de naturaleza objetiva, es decir, cuya concurrencia, cierta en cuanto a su producción (en el sí), aunque no necesariamente en cuanto al momento de su aparición (en el cuándo), es ajena a la decisión o voluntad de las partes (STJUE de 5 de febrero de 2015, Nisttabuz, C-117/14), con exclusión, por tanto, de los contratos sometidos a condiciones resolutorias de incierta producción (inciertas en el sí y en el cuándo).

No se prejuzga o predetermina, sin embargo, el régimen de contratación temporal existente en cada ordenamiento interno, sea libre o causal u objetivo, aun cuando, como se precisa en la consideración general 7 del Acuerdo marco anexo a la Directiva, «la utilización de contratos de trabajo de duración determinada basados en razones objetivas es una forma de evitar abusos». Y tampoco se prejuzga el modo de determinación formal de la duración, vigencia o temporalidad del contrato: a tra- 
vés de un plazo o un término (STJUE de 3 de julio de 2014, Fiamingo, C-363 y 407/2013) ${ }^{8}$.

Como se deduce del primer enunciado anteriormente transcrito de la cláusula 2, la Directiva no resulta aplicable a los trabajadores puestos a disposición de las empresas usuarias por parte de empresas de trabajo temporal. Ni a la relación laboral de duración determinada entre el trabajador cedido y la empresa de trabajo temporal, ni a la prestación o relación entre dicho trabajador y la empresa usuaria (STJUE de 11 de abril de 2013, Della Rocca, C-290/12).

Por otra parte, se permite que los Estados, en los actos de transposición y previa consulta con los interlocutores sociales, puedan excluir también la aplicación de la Directiva respecto de los contratos temporales de formación profesional inicial o de aprendizaje, así como de los vinculados a programas públicos de formación, inserción y reconversión profesionales, entre los que se incluyen los celebrados por la Administración con personas desempleadas e inscritas en listas de movilidad o colocación, para desarrollar durante un periodo determinado trabajos socialmente útiles o de utilidad pública (STJUE de 15 de marzo de 2012, Sibilio, C-157/11).

Fuera de estas posibles exclusiones, el ámbito de aplicación de la Directiva es omnicomprensivo, con independencia de la duración del contrato o de su ocasionalidad (STJUE de 22 de abril de 2010, Zentralbetriebsrat der Landeskrankenhäuser Tirols, C- 486/08) y del sector de actividad de que se trate. Incluso en aquellos sectores en los que se constata una especial incidencia de la necesidad de trabajo temporal por el elevado volumen de plantilla, y, consecuentemente, de la mayor frecuencia de sustituciones o interinidades, o por la variabilidad de la actividad, como la enseñanza, la sanidad o los trabajos estacionales o de campaña (STJUE de 26 de noviembre de 2014, Mascolo, C-22, 61 a 63 y 418/13; STJUE de 14 de septiembre de 2016, Pérez López, C-16/15, y ATJUE de 21 de septiembre de 2016, Popescu, C-614/15). Sin perjuicio de que en tales casos se puedan adoptar, excepcionalmente, medidas particulares frente al abuso en el encadenamiento de contratos, y hasta tratamientos diferenciados respecto de la contratación indefinida, siempre que concurran razones objetivas, pero no la exclusión del ámbito de aplicación de la norma y el Acuerdo marco comunitarios (STJUE de 3 de julio de 2014, Fiamingo, C-363 y 407/2013, y STJUE de 8 de septiembre de 2011, Rosado Santana, C-177/10). Por lo demás, la aplicación extensiva de

${ }^{8} \ll \mathrm{El}$ Acuerdo marco no contiene ninguna disposición relativa a los conceptos formales que deben figurar en los contratos de trabajo de duración determinada». 
la Directiva se ve reforzada en relación con el principio de igualdad y no discriminación, como principio general del Derecho de la Unión que se vería desvirtuado en caso de permitirse exclusiones genéricas o excesivas (STJUE de 22 de diciembre de 2010, Gavieiro, C-444/09 y C-456/09).

La Directiva alcanza igualmente a los trabajadores temporales de la Administración o del sector público (STJCE de 4 de julio de 2006, Adeneler, C-212/04, y STJCE de 7 de septiembre de 2006, Marrosu, C-53/04). De hecho, y teniendo en cuenta que el empleo temporal es mucho más numeroso en el sector privado, tanto en términos absolutos como relativos o porcentuales, respecto del conjunto de los trabajadores ${ }^{9}$, llama la atención, como se dijo, que prácticamente todas las resoluciones judiciales comunitarias se refieran a empleados del sector público. Quizá como consecuencia de la mayor protección jurídica, individual y colectiva, y, por tanto, del menor temor al planteamiento de reclamaciones o demandas judiciales de los empleados públicos, y también, destacadamente, de la más prolongada duración de sus contratos en el sector público, paradigmáticamente en España ${ }^{10}$, con el consiguiente refuerzo de sus expectativas de estabilidad y de su mayor comparabilidad e igualdad respecto de los trabajadores indefinidos ${ }^{11}$. Amén de que la mayoría de los ordenamientos cuestionados contemplan reglas o prácticas diferentes respecto de ellos en relación con las aplicables a los trabajadores temporales del sector privado.

Además, la Directiva se considera aplicable con independencia del régimen contractual-laboral o administrativo-funcionarial del empleado temporal, pues se trata, en todo caso, de personal vinculado a un empleador con un contrato o una relación de trabajo de carácter temporal o de duración determinada. Particularmente en el caso español, se incluye a los

9 En el tercer trimestre del año 2016, la Encuesta de Población Activa elaborada por el Instituto Nacional de Estadística consignaba 3.465.600 trabajadores temporales en el sector privado (27,9 por 100 del total de trabajadores de ese sector), frente a 686.800 en el sector público (22,9 por 100 del total de trabajadores de ese sector).

${ }_{10}$ A partir de los datos de la Encuesta de Población Activa elaborada por el Instituto Nacional de Estadística correspondiente al tercer trimestre del año 2016 se desprende que los contratos temporales de más de tres años suponen el 11,05 por 100 de la contratación temporal en el sector público, mientras que en el sector privado sólo llegan al 3,3 por 100; los contratos de entre dos y tres años alcanzan el 1,64 por 100 frente al 0,89 por 100, y entre uno y dos años, el 14,02 por 100 frente al 7,46 por 100. Es decir, el 26,71 por 100 de los trabajadores temporales del sector público tiene contratos superiores a un año, mientras que ese porcentaje sólo llega al 11,65 por 100 en el sector privado.

${ }^{11}$ En los asuntos provenientes de España, la duración de las relaciones temporales de las trabajadoras y los trabajadores partes en los correspondientes procesos van desde casi tres años (STJUE de 14 de septiembre de 2016, Martínez Andrés, C-184/15) hasta treinta y dos (STJUE de 9 de julio de 2015, Regojo, C-177/14). 
funcionarios temporales o interinos (STJUE de 22 de diciembre de 2010, Gavieiro, C-444/09 y C-456/09, y ATJUE de 21 de septiembre de 2016, Álvarez Santirso, C-631/15). E incluso al personal eventual de la Administración (STJUE de 9 de julio de 2015, Regojo, C-177/14), pese a tratarse de puestos de confianza o asesoramiento especial de nombramiento y cese libres, y vinculados a la autoridad que los nombra (art. 12 EBEP).

De manera más discutible, también se ha considerado incluido en el ámbito de aplicación de la Directiva a los especiales contratos de trabajo indefinidos no fijos del sector público. Creación de nuestro Tribunal Supremo de los años noventa del siglo xx destinada a cohonestar la sanción laboral interna frente a la contratación laboral irregular o fraudulenta, constituida por la presunción de contrato de trabajo ordinario de carácter fijo (arts. 8.1 y 15.3 ET) con los principios constitucionales de igualdad, mérito y capacidad en el acceso al empleo público estable (art. $103 \mathrm{CE})^{12}$, puesto que, a juicio del TJUE, quizá haciendo suyos, tácitamente, los argumentos del juzgado que plantea la petición prejudicial ${ }^{13}$, esta conversión judicial de un contrato temporal en uno indefinido no fijo «es una sanción por el recurso abusivo a sucesivos contratos de trabajo de duración determinada y no modifica la propia naturaleza de estos contratos» (ATJUE de 11 de diciembre de 2014, León Medialdea, C-86/14).

Por lo demás, en algunos casos también se ha considerado aplicable la Directiva a trabajadores tras la adquisición de la condición de indefinidos o fijos, si bien en relación con el cómputo o consideración de los periodos de trabajo temporal previos, particularmente a efectos de antigüedad o de promoción (STJCE de 13 de septiembre de 2007, Cerro Alonso, C-307/05, y STJUE de 18 de octubre de 2012, Valenza, C-302 a 305/11). Aunque, por el contrario, no se estimó aplicable al «"contrato de trabajo por tiempo indefinido de apoyo a los emprendedores" previsto por el Derecho español [puesto que] no es un contrato de duración determinada», teniendo en cuenta que «el periodo de prueba sirve fundamentalmente para comprobar la aptitud y la capacidad del trabajador, mientras que el contrato de trabajo de duración determinada se utiliza cuando el final del contrato de

${ }_{12}$ SSTS de 10 de diciembre de 1996 (rec. 1989/1995), 30 de diciembre de 1996 (rec. 637/1996), 11 de marzo de 1997 (rec. 3940/1996), 14 de marzo de 1997 (rec. 2974/1996) o 20 de marzo de 1997 (rec. 3305/1996), entre otras muchas.

13 «Dicho juzgado alega que, en el supuesto de tal conversión, por un lado, la Administración Pública elude la aplicación del Derecho común, ya que no está obligada a abonar una indemnización por la extinción de la relación laboral, y, por otro, esta Administración puede decidir unilateralmente extinguir esa relación». 
trabajo o de la relación laboral viene determinado por condiciones objetivas» (STJUE de 5 de febrero de 2015, Nisttabuz, C-117/14) ${ }^{14}$.

\section{PREVENCIÓN DEL ABUSO EN LA SUCESIÓN DE CONTRATOS TEMPORALES}

La cláusula 5 del Acuerdo marco anexo a la Directiva 1999/70/CE contempla un conjunto de medidas destinadas a prevenir y evitar la utilización sucesiva de los contratos de duración determinada por parte de las empresas, aunque, a diferencia de la cláusula 4 , relativa a los principios de igualdad y no discriminación de los trabajadores temporales, no es lo suficientemente incondicional y precisa para que pueda alegarse directamente ante los órganos judiciales nacionales, sin perjuicio de que éstos deban interpretar el ordenamiento interno del modo más acorde con su finalidad (STJUE de 10 de marzo de 2011, Lufthansa, C-109/09).

Debe subrayarse que, frente a otros posibles abusos y usos desviados o fraudulentos de la contratación temporal, la Directiva se limita a intentar evitar tan sólo el constituido por su utilización sucesiva o encadenada en relación con un mismo trabajador, con la finalidad de evitar la precarización de los trabajadores asalariados (STJCE de 4 de julio de 2006, Adeneler, C-212/04). No se aplica, por tanto, a los trabajadores que hayan celebrado un primer o único contrato de trabajo de duración determinada, ni siquiera cuando se utilice para cubrir necesidades permanentes de las empresas (STJCE de 23 de abril de 2009, Angelidaki, C-378/07, y STJUE de 3 de julio de 2014, Fiamingo, C-363 y 407/2013), y, en consecuencia, tampoco en supuestos de encadenamiento de contratos temporales con distintos trabajadores.

De este modo, la Directiva respeta el régimen de contratación temporal inicial existente en cada ordenamiento interno, sea libre o causal, aun cuando se reconozca que su utilización por razones objetivas es una forma de evitar abusos (consideración general séptima) ${ }^{15}$ y tan sólo limita su prolongación o sucesión en el tiempo, imponiendo a los Estados, previa consulta con los interlocutores sociales y teniendo en cuenta las necesida-

${ }^{14}$ Este polémico contrato, con un periodo de prueba de un año en todo caso, fue creado por la Ley 3/2012, de 6 de julio, de Medidas Urgentes para la Reforma del Mercado Laboral, y su inconstitucionalidad fue desestimada por STC 8/2015, de 22 de enero.

15 STJCE de 4 de julio de 2006, Adeneler, C-212/04; ATJCE de 12 de junio de 2008, Vassilakis, C-364/07, y STJCE de 23 de abril de 2009, Angelidaki, C-378/07. 
des de los distintos sectores y profesiones, la obligación de adoptar una o varias medidas preventivas con ese objeto. Al efecto concreta tres posibles: el establecimiento de razones objetivas para la renovación o prórroga de los contratos temporales, la determinación de una duración máxima total (incluida su sucesión) o la fijación de un número limitado de renovaciones posibles, de las que deberá implementarse al menos una, siempre que el Estado no cuente con medidas internas equivalentes preexistentes. No obstante, a efectos de determinar el grado de cumplimiento de los objetivos de la Directiva por parte de los Estados, y, en particular, el de las medidas preventivas equivalentes o sancionadoras frente a los abusos, también se consideran las reglas relativas a la contratación temporal inicial o única (STJCE de 23 de abril de 2009, Angelidaki, C-378/07).

Por otra parte, y a estos efectos, también se dispone que los Estados determinarán en qué condiciones los contratos temporales se entenderán sucesivos y celebrados por tiempo indefinido. A este respecto se ha estimado que se opone a la Directiva la consideración como contratos sucesivos tan sólo de aquellos separados por intervalos de un máximo de veinte días, puesto que con ello puede comprometerse el objeto, la finalidad y el efecto útil del Acuerdo marco (STJCE de 4 de julio de 2006, Adeneler, C-212/04). No así cuando se exige que dicho intervalo sea inferior a tres meses (ATJCE de 12 de junio de 2008, Vassilakis, C-364/07) o a sesenta días (STJUE de 3 de julio de 2014, Fiamingo, C-363 y 407/2013), de modo que se considere como nuevo contrato y no como sucesivo el celebrado tras haber transcurrido ese plazo desde uno anterior, siempre que, atendiendo a las circunstancias concurrentes, con ello no se pretenda eludir la protección que concede el Acuerdo marco contra los abusos, dejando transcurrir el plazo al finalizar cada contrato temporal.

En lo que se refiere a las medidas previstas en la Directiva para prevenir y evitar la contratación temporal sucesiva, se impone a los Estados la adopción al menos de una de ellas, siempre que, como se dijo, no cuente con otras equivalentes preexistentes, en cuyo caso parece que deberá de implementar alguna de aquéllas y no otras análogas o equivalentes (STJCE de 23 de abril de 2009, Angelidaki, C-378/07). Por otra parte, los Estados podrán aplicar medidas diferentes en unos u otros sectores de actividad o para distintas categorías de trabajadores, paradigmáticamente en el empleo público (ATJUE de 24 de abril de 2009, Koukou, C-519-08), sin que se imponga, al efecto, su igualdad o uniformidad, siempre que, en cualquier caso y ámbito, cuenten con alguna y sea eficiente para el cumplimiento de los objetivos de la Directiva (STJCE de 4 de 
julio de 2006, Adeneler, C-212/04, y STJUE de 14 de septiembre de 2016, Martinez Andrés, C-184/15) ${ }^{16}$.

La medida cuestionada en más ocasiones es la relativa al establecimiento de «razones objetivas que justifiquen la renovación» de los contratos temporales [cláusula 5.1.a)], para lo que no basta la simple admisión o una previsión genérica y abstracta en una norma en relación con un completo sector de actividad (ATJUE de 23 de noviembre de 2009, Lagoudakis, C-162/08, y STJUE de 26 de febrero de 2015, Comisión Europea contra Gran Ducado de Luxemburgo, C-238/14) ${ }^{17}$. Por el contrario, se exige una justificación concreta derivada de la actividad de que se trate y de sus condiciones de desarrollo o de legítimas opciones de política social, por ejemplo, en materia de fomento del empleo o de conciliación de la vida familiar (STJCE de 22 de noviembre de 2005, Mangold, C-144/04, y STJUE de 26 de noviembre de 2014, Mascolo, C-22, 61 a 63 y 418/13) ${ }^{18}$. Además, transparente, de modo que permita el enjuiciamiento o verificación de su proporcionalidad, en el sentido de responder efectivamente a una necesidad real, de permitir alcanzar el objetivo perseguido y de resultar indispensable a esos efectos (STJCE de 4 de julio de 2006, Adeneler, C-212/04, y STJUE de 23 de abril de 2009, Angelidaki, C-378/07) ${ }^{19}$. Sin que, por lo demás, se admitan razones puramente presupuestarias, que podrán justificar la adaptación de las medidas de prevención y su alcance, pero no su exclusión (STJUE de 21 de septiembre de 2016, Popescu, C-614/15).

Entre dichas razones objetivas se han admitido las necesidades de trabajo temporal con carácter general (STJCE de 23 de abril de 2009, Angelidaki, C-378/07) y, específicamente, de las derivadas de la sustitución de trabajadores o trabajadoras ausentes temporalmente (particularmente por

16 También las SSTJCE de 7 de septiembre de 2006, Vasallo, C-180/04, y Marrosu, C-53/04, y el ATJUE de 23 de noviembre de 2009, Lagoudakis, C-162/08.

17 También la STJUE de 14 de septiembre de 2016, Pérez López, C-16/15.

18 «El concepto de "razones objetivas" se refiere a las circunstancias específicas y concretas que caracterizan una determinada actividad y que, por tanto, pueden justificar en ese contexto particular la utilización sucesiva de contratos de trabajo de duración determinada [...] Tales circunstancias pueden tener su origen en la especial naturaleza de las tareas para cuya realización se celebran tales contratos y en las características inherentes a las mismas o, eventualmente, en la persecución de un objetivo legítimo de política social por parte de un Estado miembro [...] En cambio, no cumpliría los requisitos especificados en los dos apartados anteriores una disposición nacional que se limitase a autorizar la utilización sucesiva de contratos de trabajo de duración determinada de un modo general y abstracto a través de una norma legal o reglamentaria» (STJCE de 4 de julio de 2006, Adeneler, C-212/04). También el ATJCE de 12 de junio de 2008, Vassilakis, C-364/07; la STJCE de 23 de abril de 2009, Angelidaki, C-378/07, y el ATJUE de 24 de abril de 2009, Koukou, C-519-08, entre otras resoluciones.

19 También la STJUE de 26 de noviembre de 2014, Mascolo, C-22, 61 a 63 y 418/13. 
motivos familiares y de maternidad y paternidad), de la cobertura interina de puestos durante los procesos de selección en el sector público, siempre que estén delimitados (STJUE de 26 de noviembre de 2014, Mascolo, C-22, 61 a 63 y 418/13), o de la variabilidad o coyunturalidad de la actividad (enseñanza, sanidad o campañas) ${ }^{20}$. Incluso cuando estas necesidades se produzcan con carácter recurrente o permanente y, en consecuencia, puedan también ser cubiertas con personal indefinido. Aun cuando, en tales casos, deba comprobarse que la contratación se realiza por necesidades provisionales y no permanentes, atendiendo a cada supuesto concreto y a las circunstancias concurrentes, tales como el número de contratos realizados o la duración de las relaciones temporales (STJUE de 26 de enero de 2012, Kücük, C-586/1), en su caso también mediante prueba estadística y global en el sector o actividad de que se trate (STJUE de 26 de noviembre de 2014, Mascolo, C-22, 61 a 63 y 418/13, y STJUE de 14 de septiembre de 2016, Pérez López, C-16/15).

En cuanto a las otras dos medidas previstas en la cláusula 5 de la Directiva, sobre el establecimiento de «una duración máxima total de sucesivos contratos» temporales o un «número de renovaciones», no parece que su superación tenga que conllevar en todo caso la transformación del contrato en indefinido (STJCE de 23 de abril de 2009, Angelidaki, C-378/07) ${ }^{21}$, aunque esta consecuencia resulte acorde con la finalidad perseguida. Claro está, siempre que tales excesos tengan otras consecuencias igualmente preventivas o sancionadoras y protectoras de los trabajadores afectados (STJCE de 4 de julio de 2006, Adeneler, C-212/04), por ejemplo, mediante su resarcimiento o indemnización (SSTJCE de 7 de septiembre de 2006, Vasallo, C-180/04, y Marrosu, C-53/04) 22, y sean efectivas y no limitativas en la práctica (ATJCE de 12 de diciembre de 2013, Papalia, C-50/13), también desde el punto de vista procesal (STJUE de 14 de septiembre de 2016, Martínez Andrés, C-184/15).

En cualquier caso, para que la transformación del contrato o contratos temporales irregulares o sucesivos en una relación indefinida sea

${ }^{20}$ La STJUE de 13 de marzo de 2014, Márquez Somohano, C-190/13, parece aceptar la colaboración docente de profesionales externos como profesores asociados de universidad temporal y a tiempo parcial (art. 53 LOU) como causa objetiva que justifica la inaplicación de los límites y las medidas contra el abuso en la sucesión de contratos temporales, teniendo en cuenta, además, que con ello no se precariza su situación, dado que deben tener una actividad profesional externa, pero siempre que no se utilicen para actividades permanentes.

${ }_{21}$ También la STJCE de 7 de septiembre de 2006, Marrosu, C-53/04, y el ATJCE de 12 de junio de 2008, Vassilakis, C-364/07.

${ }^{22}$ También el ATJUE de 1 de octubre de 2010, Affatato, C-3/10. 
acorde con la Directiva deberá conllevar unas consecuencias protectoras análogas a las generales de estas relaciones, paradigmáticamente en materia de extinción contractual (ATJUE de 11 de diciembre de 2014, León Medialdea, C-86/14) ${ }^{23}$, y no podrá implicar una modificación sustancial de las condiciones de trabajo previas de carácter globalmente desfavorable (STJUE de 8 de marzo de 2012, Huet, C-251-11).

En fin, como medidas equivalentes para prevenir los abusos derivados de la utilización de sucesivos contratos o relaciones laborales de duración determinada se ha aceptado la transformación de un contrato de trabajo de duración determinada en contrato por tiempo indefinido cuando atiende, en realidad, necesidades permanentes y duraderas del empleador (STJCE de 23 de abril de 2009, Angelidaki, C-378/07), y, más matizadamente, el establecimiento de indemnizaciones o sanciones (ATJUE de 24 de abril de 2009, Koukou, C-519-08).

A este respecto, no es claro que las posibles sanciones puedan considerarse propiamente como medidas equivalentes a las preventivas previstas en la cláusula 5 de la Directiva, puesto que aquéllas no se contemplan en la norma comunitaria, son reactivas frente al abuso ya constatado y se aplican como medio de protección respecto del trabajador perjudicado (STJUE de 21 de septiembre de 2016, Popescu, C-614/15). Aun cuando, en último término, también tengan una función y efectos disuasorios y, por ende, preventivos. En cualquier caso, y respecto de tales sanciones específicas frente a los abusos no establecidas en la Directiva y que deberán adoptarse de manera autónoma por los Estados, tendrán que ser proporcionadas, efectivas y disuasorias, y no podrán ser «menos favorables que las aplicables a situaciones similares de carácter interno (principio de equivalencia) ni hacer imposible en la práctica o excesivamente difícil el ejercicio de los derechos conferidos por el ordenamiento jurídico de la Unión (principio de efectividad)» (STJUE de 3 de julio de 2014, Fiamingo, C-363 y 407/2013, y ATJUE de 21 de septiembre de 2016, Popescu, C-614/15) $)^{24}$.

${ }^{23}$ En relación con el contrato indefinido no fijo creado por la jurisprudencia española. Vid. también la STJUE de 14 de septiembre de 2016, Martínez Andrés, C-184/15.

${ }^{24}$ «Cuando se ha producido una utilización abusiva de contratos de trabajo de duración determinada sucesivos es indispensable poder aplicar alguna medida que ofrezca garantías de protección de los trabajadores efectivas y equivalentes, con objeto de sancionar debidamente dicho abuso y eliminar las consecuencias de la violación del Derecho de la Unión». También las SSTJCE de 4 de julio de 2006, Adeneler, C-212/04; 7 de septiembre de 2006, Vasallo, C-180/04, y Marrosu, C-53/04, y 14 de septiembre de 2016, Pérez López, C-16/15. 


\section{IGUALDAD Y NO DISCRIMINACIÓN DE LOS TRABAJADORES TEMPORALES}

Las reglas de aplicación del principio de igualdad y no discriminación de la Directiva 70/1999/CE parten de la doble caracterización del trabajo temporal como trabajo flexible y precario, y, en algún momento y país, feminizado. Lo primero implica que el trabajador, ligado de forma más frágil e inestable a la empresa, tiene una menor fuerza contractual de cara a la negociación y aceptación de sus condiciones de trabajo, o incluso a la reclamación de sus derechos, al estar condicionado por las expectativas de renovación, prórroga o conversión en fijo, y un estatus laboral inferior como consecuencia de la vinculación de ciertos derechos a la permanencia en la empresa (antigüedad, vacaciones, indemnizaciones por extinción, pensiones, etc.). Lo segundo supone que el trabajo temporal pueda constituir también una causa de discriminación indirecta por razón del sexo, cuando no se trate de una condición neutra desde ese punto de vista, lo que contribuye a fortalecer el mandato de igualdad y no discriminación de los trabajadores y trabajadoras temporales ${ }^{25}$.

Desde esta doble dimensión, la cláusula 4 del Acuerdo marco dispone que los trabajadores temporales no podrán ser tratados de manera menos favorable que los trabajadores fijos comparables (del mismo centro, puesto y trabajo) o peor que conforme a lo establecido con carácter general en el convenio aplicable, en la negociación colectiva o en la práctica nacional por el simple hecho de tener un contrato de duración determinada, salvo que el tratamiento diferenciado esté justificado por razones objetivas, en su caso mediante la aplicación de la regla de prorrateo temporal. $\mathrm{Y}$ de manera más específica, también se dispone su tratamiento igualitario en materia de antigüedad, a efectos de devengo de las condiciones y

${ }^{25}$ No obstante, y a diferencia del trabajo a tiempo parcial, claramente feminizado, el trabajo temporal no siempre tiene esa característica y nunca tan marcada. En este sentido, en el año 2015, y como media en los países de la Unión Europea, el 13,8 por 100 de los hombres trabajadores eran temporales (25,1 por 100 en España) frente al 14,6 por 100 de las mujeres trabajadoras (25,2 por 100 en España), y del conjunto de trabajadores temporales, las mujeres representaban el 49,51 por 100 (48,002 por 100 en España). Es decir, menos de la mitad de los trabajadores temporales europeos (y españoles) eran mujeres en 2015 (Ministerio de Empleo y Seguridad Social, Anuario de Estadísticas 2015, Encuesta de Fuerzas de Trabajo Unión Europea). Y aunque las partes en los asuntos resueltos por el Tribunal comunitario son mujeres con más frecuencia que hombres, tampoco se trata de una diferencia muy significativa con carácter general (alrededor de 19 frente a 17), aunque quizá sí en el caso de los asuntos de origen español (alrededor de 9 frente a 4). 
derechos a ella vinculados, de nuevo salvo que se justifique objetivamente un trato diferente.

Como se dijo y es fácilmente comprensible, el principio de igualdad y no discriminación de los trabajadores temporales constituye el núcleo duro de la Directiva, cuya finalidad es mejorar la calidad del trabajo temporal, evitar que se perpetúe el mantenimiento de una situación desfavorable (STJUE de 22 de diciembre de 2010, Gavieiro, C-444/09 y C-456/09) e «impedir que una relación laboral de esta naturaleza sea utilizada por un empleador para privar a dichos trabajadores de derechos reconocidos a los trabajadores con contrato de duración indefinida» (STJCE de 13 de septiembre de 2007, Cerro Alonso, C-307/05). Constituye, además, un principio general y una disposición mínima del Derecho comunitario, y, como tal, su interpretación debe hacerse de forma extensiva y no restrictiva (STJCE de 13 de septiembre de 2007, Cerro Alonso, C-307/05, y STJUE de 15 de abril de 2008, Impact, C-268/06).

Por otra parte, y a diferencia de la otra cláusula esencial del Acuerdo marco, la relativa a la prevención y evitación del abuso en la sucesión de contratos de duración determinada, la referida a la igualdad de derechos de los trabajadores temporales respecto de los fijos tiene un contenido incondicional y lo suficientemente claro para poder ser invocada por un particular ante la jurisdicción nacional, y goza de efecto directo frente a cualquier organismo público (STJUE de 12 de diciembre de 2013, Carratù, C- 361/12) ${ }^{26}$. Aunque ciertamente se trata de dos cláusulas íntimamente relacionadas, toda vez que el reconocimiento a los trabajadores temporales de los mimos derechos que a los fijos en situación comparable contribuye eficazmente a evitar la utilización abusiva de la contratación de duración determinada (ATJUE de 21 de septiembre de 2016, Álvarez Santirso, C-631/15). Amén de aplicarse comunes criterios interpretativos en relación con las razones objetivas que pueden justificar las excepciones tanto a las reglas generales de igualdad como a las de prevención de los abusos en la sucesión contractual (STJCE de 13 de septiembre de 2007, Cerro Alonso, C-307/05).

En lo que se refiere a las condiciones de trabajo a las que resulta de aplicación el principio de igualdad de trato de los trabajadores temporales,

26 «Entre las entidades a las que pueden oponerse las disposiciones de una Directiva susceptible de tener efecto directo se incluye todo organismo, con independencia de su forma jurídica, al que en virtud de un acto de la autoridad pública se le haya encomendado la prestación de un servicio de interés público bajo la tutela de dicha autoridad y que disponga a tal efecto de poderes exorbitantes respecto de las normas aplicables a las relaciones entre particulares». 
el Tribunal de Justicia ha consolidado una doctrina extensiva y casi omnicomprensiva que alcanza a todas las derivadas de la relación de trabajo o empleo, incluidas las retribuciones y las condiciones de empleo (STJUE de 12 de diciembre de 2013, Carratù, C- 361/12).

A las primeras, por entender que la expresa exclusión de las remuneraciones entre las competencias de la Unión Europea en materia de política social (art. 153.5 del TFUE-2016) se refiere a su estructura, composición y cuantía, que no pueden ser objeto de regulación comunitaria, pero no a la exigencia de los principios de igualdad y no discriminación de los trabajadores en relación con las retribuciones fijadas en los ordenamientos nacionales competentes (STJCE de 13 de septiembre de 2007, Cerro Alonso, C-307/05). Paradigmáticamente respecto de los complementos salariales por antigüedad (ATJUE de 18 de marzo de 2011, Montoya Medina, C-273/10, y STJUE de 9 de julio de 2015, Regojo, C-177/14), o por formación (ATJUE de 9 de febrero de 2012, Lorenzo Martínez, C-556/11) o evaluación (ATJUE de 21 de septiembre de 2016, Álvarez Santirso, C-631/15).

Incluso en relación con las pensiones, asimiladas a estos efectos a las remuneraciones cuando «dependen de la relación de empleo que vincula al trabajador con el empleador, con exclusión de las que se derivan de un régimen legal a cuya financiación contribuyan los trabajadores, los empleadores y, en su caso, los poderes públicos en una medida que depende menos de tal relación de trabajo que de consideraciones de política social», y sin perjuicio de la posible aplicación de la regla de pro rata temporis en su caso (STJUE de 15 de abril de 2008, Impact, C-268/06).

Y respecto de las condiciones de empleo, particularmente a las referidas a la extinción del contrato y el despido ${ }^{27}$, como el preaviso de la denuncia o comunicación de la finalización del contrato (STJUE de 13 de marzo de 2014, Nierodzik, C-38/13) o las indemnizaciones (STJUE de 12 de diciembre de 2013, Carratù, C- 361/12, y STJUE de 14 de septiembre de 2016, Diego Porras, C-596/14), o al cómputo de los servicios prestados temporalmente a efectos de promoción profesional (STJUE de 8 de septiembre de 2011, Rosado Santana, C- 177/10), o, más matizada-

${ }^{27}$ En este sentido, el despido se incluye expresamente entre las condiciones de trabajo en otras Directivas comunitarias sobre igualdad (Directiva 2000/78/CE del Consejo, de 27 de noviembre de 2000, relativa al establecimiento de un marco general para la igualdad de trato en el empleo y la ocupación, y Directiva 2006/54/CE del Parlamento Europeo y del Consejo, de 5 de julio de 2006, relativa a la aplicación del principio de igualdad de oportunidades e igualdad de trato entre hombres y mujeres en asuntos de empleo y ocupación). 
mente, de acceso al empleo fijo (STJUE de 18 de octubre de 2012, Valen$z a$, C-302 a 305/11) ${ }^{28}$.

Conforme a la cláusula 4 del Acuerdo marco, la aplicación del principio de igualdad de derechos de los trabajadores temporales está condicionada, con carácter general, a la concurrencia de una situación comparable, esto es, al reconocimiento del concreto derecho de que se trate a los trabajadores indefinidos o fijos que ocupen puestos o realicen funciones semejantes. Sin que sirva, a efectos de la Directiva, la comparación (o la diferencia de trato) entre distintos grupos o categorías de trabajadores o empleados temporales (STJUE de 14 de septiembre de 2016, Diego Porras, C-596/14), aunque sí entre empleados temporales y fijos con vínculos de naturaleza o carácter diferente, laboral y administrativo o funcionarial, siempre que la comparación se realice atendiendo a la vigencia de la relación y no a la naturaleza o carácter del vínculo (STJUE de 14 de septiembre de 2016, Pérez López, C-16/15).

Además, con frecuencia los elementos que pueden impedir la comparabilidad también pueden servir como razones objetivas para justificar determinados tratamientos diferenciados de los trabajadores temporales (STJUE de 18 de octubre de 2012, Valenza, C-302 a 305/11) ${ }^{29}$ en los términos igualmente admitidos por esta cláusula, que, como se dijo, también recuerdan a los establecidos respecto de la posible justificación de la renovación o sucesión de contratos de duración determinada de la cláusula 5.1.a).

Particularmente en lo que se refiere a la exigencia de objetividad, transparencia y proporcionalidad, debiendo tratarse, por tanto, de «elementos precisos y concretos, que caracterizan la condición de trabajo de que se trata, en el contexto específico en que se enmarca y con arreglo a criterios objetivos y transparentes, a fin de verificar si dicha desigualdad responde a una necesidad auténtica, si permite alcanzar el objetivo perseguido y si resulta indispensable al efecto» (STJCE de 13 de septiembre de 2007, Cerro Alonso, C-307/05) ${ }^{30}$. De este modo, la falta de comparabilidad

${ }^{28}$ En el mismo sentido, los AATJUE de 7 de marzo de 2013, Bertazzi, C-393-11, y 4 de septiembre de 2014, Bertazzi, C-152/14. Vid. también el ATJUE de 21 de septiembre de 2016, Álvarez Santirso, C-631/15.

${ }^{29}$ «La naturaleza de las funciones ejercidas por las recurrentes en el litigio principal durante los años en los que prestaron servicios para la AGCM en el marco de contratos de duración determinada y la calidad de la experiencia adquirida en esta condición no son sólo uno de los factores que pueden justificar objetivamente una diferencia de trato con respecto a los funcionarios de carrera. Figuran también entre los criterios que permiten comprobar si las interesadas se hallan en una situación comparable respecto de éstos».

${ }^{30}$ La STJUE de 13 de marzo de 2014, Nierodzik, C-38/13, estima que la cláusula 4.1 
o la razón del tratamiento diferenciado deberán estar justificadas objetivamente en relación con la concreta condición o derecho cuestionados, pues no todas ni las mismas podrán justificar cualesquiera diferencias de trato (STJUE de 9 de julio de 2015, Regojo, C-177/14).

En cualquier caso, parece que los tratamientos diferenciados lo serán, o podrán serlo, más en términos de adaptación de la concreta condición o derecho de que se trate en relación con el trabajo temporal, que de simple exclusión (STJUE de 22 de abril de 2010, Zentralbetriebsrat der Landeskrankenbäuser Tirols, C- 486/08) ${ }^{31}$. Y más en términos funcionales que aritméticos o de simple prorrateo temporal, aunque también se prevea expresamente este principio o regla de pro rata temporis «cuando resulte adecuado» (cláusula 4.2).

En este marco y con carácter general, las posibles diferencias de trato no pueden justificarse por su simple previsión o cobertura legal, reglamentaria o convencional, o por el mero carácter temporal de su contrato o por reflejos intrínsecos al mismo, como las expectativas de estabilidad o la previsibilidad de la finalización del contrato, no suficientemente objetiva. Y «pueden tener su origen, en particular, en la especial naturaleza de las tareas para cuya realización se celebran los contratos de duración determinada y en las características inherentes a las mismas», en «la persecución de un objetivo legítimo de política social por parte de un Estado miembro» o en los requisitos de formación (STJUE de 14 de septiembre

del Acuerdo marco «se opone a una normativa nacional, como la controvertida en el litigio principal, que prevé, para la resolución de los contratos de trabajo de duración determinada de duración superior a seis meses, la posibilidad de aplicar un plazo de preaviso fijo de dos semanas independientemente de la antigüedad del trabajador, mientras que el plazo de preaviso para la resolución de los contratos de trabajo por tiempo indefinido se determina en función de la antigüedad del trabajador y varía entre dos semanas y tres meses, cuando ambas categorías de trabajadores se encuentren en situaciones comparables». Al efecto tiene en cuenta que la aplicación del plazo de preaviso previsto en la normativa nacional para los contratos indefinidos al contrato de la trabajadora parte en este proceso habría implicado, por razón de su antigüedad, el doble de plazo de preaviso que el establecido para los contratos temporales.

31 Vid. también la STJUE de 18 de octubre de 2012, Valenza, y los AATJUE de 7 de marzo de 2013, Bertazzi, C-393-11, y 4 de septiembre de 2014, Bertazzi, C-152/14, en relación con la no consideración, en absoluto, de los periodos previos de trabajo temporal para el acceso a un proceso de estabilización en el empleo; el ATJUE de 9 de febrero de 2012, Lorenzo Martínez, C-556/11, en relación con la exclusión de un complemento retributivo por formación permanente; el ATJUE de 18 de marzo de 2011, Montoya Medina, C-273/10, en relación con el complemento de antigüedad; la STJUE de 14 de septiembre de 2016, Diego Porras, C-596/14, en relación con la indemnización por extinción involuntaria del contrato de trabajo, o el ATJUE de 21 de septiembre de 2016, Álvarez Santirso, C-631/15, en relación con un complemento retributivo derivado de la evaluación docente. 
de 2016, Diego Porras, C-596/14). En este marco, también se han aceptado como razones objetivas que podrían justificar tratamientos diferenciados, aunque en la mayoría de los casos obiter dicta, la calidad de la experiencia (STJUE de 18 de octubre de 2012, Valenza, C-302 a 305/11), la discriminación inversa de los trabajadores fijos (ATJUE de 21 de septiembre de 2016, Álvarez Santirso, C-631/15), o las condiciones de acceso y cese (STJUE de 9 de julio de 2015, Regojo, C-177/14).

Pero es significativo que en la mayoría de los asuntos en los que el Tribunal de Justicia ha valorado la adecuación de normas nacionales a la cláusula de igualdad de la Directiva 1999/70/CE, en todos, en el caso de España, se ha estimado su oposición. Quizá con la matizada excepción del derecho a la indemnización por la extinción del contrato de trabajo, cuyo tratamiento diferenciado no se estimó contrario a la Directiva en la STJUE de 12 de diciembre de 2013, Carratù, C- 361/12, y en el ATJUE de 30 de abril de 2014, D'Aniello, C-89/13, por considerarse que no concurrían situaciones comparables entre la finalización del contrato de un trabajador temporal irregular y el despido de un trabajador indefinido, pero sí en la STJUE de 14 de septiembre de 2016, Diego Porras, C-596/14, que entendió comparable la situación de un trabajador sustituto con la de un trabajador fijo. Si bien es cierto que en aquellos supuestos se trataba de regímenes indemnizatorios diferentes y en este, de la simple y completa exclusión del derecho a una indemnización por extinción del contrato temporal de sustitución. 
